\title{
La transposición didáctica interna de la Revolución Industrial en manuales de enseñanza media: textualización de las nociones de cambio y causalidad
}

\author{
Internal didactic transposition of the Industrial Revolution \\ in textbooks for 4th degree of secondary education: textualizing \\ the concepts of change and causality
}

PAÜL LIMORTI

UNIVERSITAT D'ALACANT

Recibido: 30/03/204

Aceptado: 30/04/2014

ABSTRACT: This paper analyses the pedagogical concept of didactic transposition by applying it to the case of the body of knowledge «Industrial Revolution» as textualized in a sample of five History textbooks for the 4th grade of the Spanish secondary education (ages 15-16). We pay special attention to the way notions of transformation and cause are discursively expressed. All of this in order to establish the foundations of a discursive analysis that would help to better know the grammatical structures (of a L1, L2 or LE) through whom students have access to the academic knowledge and the control of which is essential for the comprehension and production of academic texts in the field of the Social Sciences.

Keywords: didactic transposition, discourse analysis, textbooks, History textbooks, CLIL, didactics of Social Sciences, curriculum analysis.

RESUMEN: Este artículo estudia el concepto pedagógico de transposición didáctica aplicándolo al caso del saber histórico «Revolución Industrial» tal y como aparece textualizado en una muestra de cinco manuales de ciencias sociales para $4 .^{\circ}$ de Educación Secundaria. Se analiza especialmente la manera como se expresan discursivamente las nociones de transformación y de causa. Todo ello con el objetivo de sentar las bases de un análisis discursivo que permita conocer las estructuras gramaticales (de una L1, L2 o LE) a través de las cuales el alumno accede al contenido académico y cuyo dominio es vital para la comprensión y la producción de textos académicos del ámbito de las ciencias sociales. 
Palabras clave: transposición didáctica, análisis del discurso, manualística, libros de texto de historia, AICLE, CLIL, didáctica de las ciencias sociales, análisis del currículum.

\section{Transposición didáctica, currículo prescrito y currículo en acción}

El currículo prescrito y el currículo en acción serían los dos polos entre los que circula la corriente alterna de la transposición didáctica. Transposición didáctica, el concepto acuñado por Yves Chevallard (1991), parte de la existencia de un sistema didáctico compuesto por un docente y sus alumnos y un saber disciplinar, «sabio» (savant), científico, que debe convertirse en «saber enseñado». Para Chevallard habría un «espíritu» disciplinar de la materia enseñada, previo al acto de enseñar. El «saber enseñado» sería un «saber exiliado» y uno de los lugares de exilio de este saber es el libro de texto. Sobre el libro y sobre el docente recae, entonces, la autoridad académica, ellos son los agentes legitimadores del saber.

Por otro lado, el manual legitimador constituye un obstáculo contra un currículo en acción dinámico, centrado en el proceso de conocimiento, en la exploración y en el descubrimiento. En la redacción del «texto del saber»« en el texto del manual y en el discurso didáctico el conocimiento se naturaliza. Esta naturalización es el resultado de un proceso de descontextualización y recontextualización. El manual efectúa esta operación a través de la textualización del conocimiento: texto e imagen entablan una relación dinámica que constituye un nuevo contexto para el saber sabio. En este contexto o, mejor, cotexto, se concreta la desproblematización, la despersonalización y la descontextualización de los significantes. Mientras, el saber enseñado, encapsulado en su texto, caduca, envejece en relación con la sociedad, resulta superado periódicamente por la evolución del saber sabio o por la moral imperante.

El concepto de saber sabio y de transposición didáctica en su planteamiento deductivo, de arriba a abajo, establece una relación dialéctica con la idea de currículo en acción de autores como Stenhouse. En el currículo en acción el profesor guía a sus alumnos en el descubrimiento de un conocimiento no cerrado, problemático, que hay que construir yendo a sus fuentes y a través de la práctica, es decir, en un discurrir inductivo, de abajo a arriba. Chevallard considera una pretensión desaforada pensar que el conocimiento sea algo que no venga dado de una vez por 
todas y que pueda ser «construido» desde «abajo», desde el aula. Stenhouse, aun admitiendo que la deformación que el acto de enseñar impone a la materia enseñada (el saber sabio) es inevitable («Toda enseñanza falsifica su materia cuando la moldea en la forma de la enseñanza y del aprendizaje» (Stenhouse, 1985: 152), propone que se minimice esa falsificación del conocimiento a través de lo que denomina «arte de la pedagogía». Ese arte es el método que construye el currículo en acción y el análisis del discursos puede contribuir a perfeccionarlo, como veremos.

Habría en el proceso de transposición didáctica dos fases, una primera transposición externa mediante la cual el saber sabio se selecciona y se prescribe en forma de decretos curriculares, aparece así el currículo legislado; es el proceso que Gimeno Sacristán denomina «organización del saber dentro de la escolaridad» (1988: 134). A partir de este momento se inicia la transposición didáctica interna, la textualización en forma de manual, de material curricular o de discurso docente. En palabras de Bronckart y Plazaola: «Las propiedades de los contenidos didactizados son ampliamente determinadas por las de los textos o discursos que los instauran» (2000: 46). La cuestión ya había sido puesta de manifiesto por John Dewey (1925) mucho antes y constituye un motivo recurrente en las distintas corrientes de renovación pedagógica: aprender en la escuela tradicional significa la adquisición de aquello que ya se encuentra incorporado en los libros y en las cabezas de los profesores, lo que se enseña es un saber estático, un producto acabado, cerrado, naturalizado. Un producto textual.

El saber histórico se descontextualiza respecto de sus fuentes académicas y al mismo tiempo se muestra en el manual lejos del entorno inmediato del alumno. Entre los manuales examinados, solo el de la editorial Castellnou intenta contextualizar el saber enseñado en su origen «sabio» cuando explica el origen académico del término «revolución industrial». Por otra parte, en ninguno de los cinco manuales estudiados, a pesar de venderse como adaptaciones del currículo de la Comunidad Valenciana, hay referencias al caso arquetípico, y bien estudiado por los historiadores valencianos, de la revolución industrial producida en Alcoi en pleno siglo XIX, solamente el manual de Voramar/Santillana dedica un apartado a la industrialización valenciana. Tampoco, por supuesto, se enlaza con la revolución industrial tardía, pero significativa para los alumnos valencianos, que se produjo en el siglo xx: la industrialización de Elda o de Elx (Alicante) a través de la modernización de la industria de las alpargatas no se trata en ninguno de los libros. ${ }^{1}$ Es tarea de los profesores convertir el

1. Esta baja presencia de los elementos históricos propios de las comunidades autónomas en los libros de historia, por debajo incluso del mínimo marcado en los currículos oficiales, ya 
«saber prescrito» en el decreto curricular (primera transposición didáctica) y que aparece textualizado en el manual (el «saber a enseñar» fruto de la segunda transposición didáctica), en «saber efectivamente enseñado», saber recontextualizado que vuelve de abajo a arriba. Este es el caso de la unidad didáctica diseñada y llevada a la práctica por la profesora del IES Valle de Elda, Irina Agulló: «Elda la bien calzada», dentro del Proyecto ComBas del Centro Nacional de Innovación e Investigación Educativa del Ministerio de Educación, un caso paradigmático de tercera transposición didáctica en el ámbito del aula, o currículo en acción.

Así pues, el estudio de la textualización del saber sabio, tanto en los materiales curriculares, como en el discurso docente, es una vía de investigación de gran interés, tanto para el análisis del discurso como para la didáctica general y específica. Para llevarla a cabo habría que analizar de manera pormenorizada los géneros discursivos que se utilizan en las diferentes esferas de producción de los saberes. Es decir, habría que examinar la forma de organización del saber sabio (u «objeto de discurso», según Bronckart y Plazaola (2000)) en el marco textual y contextual en el cual se integra. En nuestro caso el objeto de discurso es la Revolución Industrial, el género discursivo es el manual escolar de historia y el contexto el aula de $4 .^{\circ}$ curso de Educación Secundaria.

\section{La transposición didáctica en los libros de historia}

Rafael Valls detalla los puntos de estudio que tendría que abordar cualquier investigación sobre la transposición didáctica en los libros de historia:

- la actualización científica y una imagen adecuada de la historiografía postpositivista: distinción entre datos, hipótesis, interpretaciones y valoraciones; carácter procesual, multifactorial-causal y multiperspectivo del conocimiento histórico; otreidad y «presencia» del pasado; los documentos, como testimonios y como «discursos-representaciones»,

- grado de adecuación de la presentación y de los tratamientos realizados a unos niveles científicamente aceptables: grado de distorsión, simplificación, reducción o fragmentación de los análisis realizados; grado de interrelaciónintegración-multicausalidad de los factores explicativos; grado de compara-

fue puesta de manifiesto por Jordi Burguera (2002), a propósito de los manuales de historia catalanes. El estudio de Burguera era una respuesta, basada en datos empíricos, a la polémica desatada por un informe de la Real Academia de la Historia sobre el tratamiento de la «historia de España» en los manuales de las comunidades autónomas con lengua propia. 
tivismo introducido en el tratamiento de los fenómenos históricos analizados tanto en su escala geográfico-espacial como temporal (uso de ejemplos y contraejemplos, que evitasen las trampas y falseamientos de una historiografía sectaria); análisis de los conceptos utilizados, de sus connotaciones y de su carga valorativa implícita. (Valls, 2008: 509-510)

Olvida Valls en esta pormenorizada lista de puntos conflictivos de la transposición didáctica del saber histórico, los procesos de textualización, que al fín y al cabo son los que en última instancia determinarán el grado de comprensibilidad de texto escolar. ¿Qué estructuras lingüísticas utilizan los autores de los manuales para expresar las «valoraciones», los «procesos», las «causas», las «explicaciones», las «comparaciones», los «análisis», las «transformaciones», la expresión del tiempo y del espacio...? Esta es la parte que se reserva el análisis del discurso en el estudio de la transposición didáctica del saber histórico en los manuales de historia: el estudio de las estructuras lingüísticas implicadas en la textualización del saber sabio.

En el caso que nos ocupa, el del saber sabio «Revolución Industrial», la primera transposición didáctica la realizan los decretos curriculares, que constituyen el currículo prescrito. Los manuales analizados se ajustan al currículo de la Comunidad Valenciana que a su vez concreta el decreto ministerial de mínimos. Los manuales concretan, pues, el contenido prescrito en los decretos para cuarto curso de Educación Secundaria Obligatoria. El objetivo de etapa para la asignatura Ciencias sociales, Geografía e Historia es: "Conocer los aspectos fundamentales de la cultura, la geografía y la historia de la Comunitat Valenciana, de España y del mundo; respetar el patrimonio artístico, cultural y lingüístico; conocer la diversidad de culturas y sociedades a fin de poder valorarlas críticamente y desarrollar actitudes de respeto por la cultura propia y por la de los demás».

El currículo establece además el marco en que los contenidos históricos (el «saber sabio»), se integran: «En cuarto de ESO se sintetizan las bases históricas de la sociedad actual y las transformaciones económicas, políticas y sociales producidas desde el siglo XVIII hasta la actualidad. Se hace, además, hincapié en la historia contemporánea española, singularmente en la configuración del Estado democrático en España y en su pertenencia a la Unión Europea».

Los contenidos prescritos para cuarto corresponden al «Bloque 2: Bases históricas de la sociedad actual»:

- El auge de la revolución industrial. Transformaciones económicas y tecnológicas. La expansión económica valenciana de finales del siglo XIX. 
- La nueva sociedad: viejas y nuevas élites; las clases populares. Formas de vida en la ciudad industrial.

Por otra parte, el criterio de evaluación relacionado con este contenido es el número 5: «Comprender las transformaciones socioeconómicas de la Revolución industrial, así como los acontecimientos más relevantes que explican el protagonismo de Europa durante la época del imperialismo, sus consecuencias y su declive». A este criterio se podría añadir el número 11, que define procesos cognitivos relacionados con la comprensión de procesos históricos o «transformaciones»: «Identificar las causas y consecuencias de hechos y procesos históricos significativos, establecer conexiones entre ellas y reconocer la causalidad múltiple que comportan los hechos sociales».

La palabra «síntesis» («En cuarto de ESO se sintetizan...») nos muestra uno de los procesos de transposición didáctica: la simplificación. La Revolución Industrial se presenta como una «transformación», un proceso de cambio en el tiempo, que afecta aspectos económicos, políticos y sociales, y que se considera uno de los fundamentos históricos de las sociedades actuales. «Proceso» $\mathrm{y}$ «transformación», junto a «causa» $\mathrm{y}$ «consecuencia» serían las nociones determinantes en la textualización de este contenido del saber histórico en la segunda transposición didáctica. Analizaremos, pues, la transposición didáctica de las nociones de «proceso», «transformación» y «causa-efecto», en el discurso de una muestra cinco manuales de historia de $4^{\circ}$ de ESO.

\section{La expresión lingüística de las nociones de «proceso», «transformación» y «causa-efecto»}

Como hemos apuntado con anterioridad, el material curricular impreso es un elemento mediador entre el alumno y el saber histórico, debe ayudarle a acceder al contenido curricular prescrito y debería estimular procesos cognitivos estrechamente vinculados con la manera como se comprende este tipo de contenido: la comprensión de los procesos, es decir, comprender la causalidad.

En este sentido, Blanco Rebollo (2008: 58) establece una división en tres grados de los conceptos temporales asociados al saber histórico a los que debe enfrentarse un alumno de Educación Secundaria: nivel bajo (datación, sucesión y simultaneidad); nivel medio (permanencia, secuenciación, continuidad, cambio y empatía); y nivel alto (duración, periodización y causalidad). Nuestros alumnos de $4 .^{\circ}$ de ESO deberían ser capaces, según este baremo, de enfrentarse a los conceptos situados en el nivel alto; estas serían, según el autor, «operacio- 
nes mentales especiales propias de la investigación» que requieren una transposición didáctica compleja, un trabajo específico por parte del profesor y un adecuado tratamiento pedagógico de los libros de texto. De las nociones apuntadas por Blanco Rebollo nos interesan las nociones de «cambio» («acontecimientos o situaciones que han sufrido una transformación en el tiempo y han provocado el nacimiento de nuevos sistemas»), nivel medio; y «causalidad» («estudio de los motivos que explican el origen de un proceso o un hecho histórico»), nivel alto, y de especial dificultad didáctica, según el autor.

\subsection{Nominalización}

La nominalización, en tanto que proceso semántico de abstracción y conceptualización, es uno de los principales obstáculos que deben superar los discentes en su camino académico (Salvador, 2005: 76). El uso de la nominalización (sustantivos deverbales) es constante en todos los manuales para referirse a los diferentes aspectos del macroproceso de «transformación» histórica conocido como Revolución Industrial: «aplicación», «expansión», «ampliación», «uso», «aparición», «aumento», «disminución», «crecimiento», «mejora», «producción», «avance», «incremento».

\subsubsection{Cadenas léxicas}

A veces el verbo sustantivado constituye el núcleo de nominalización semántica del cual parte una cadena léxica que se aproxima a una unidad fraseológica o a una colocación por su grado de recurrencia (Salvador, 2005: 81-82): «progresos técnicos», «aumento demográfico».

Estas cadenas constituyen muchas veces el título de los epígrafes de los apartados y sirven de guía para el alumno durante la lectura del manual. De este modo la categorización y la clasificación de los «cambios» y de las «transformaciones» se resaltan gráficamente en el libro texto: «Los cambios demográficos», «Las transformaciones económicas» [A]; «Los cambios tecnológicos» [C]; «La modernización agrícola» [C]; «Las innovaciones de la industria textil», «Las innovaciones de la industria siderúrgica» [C]; «El crecimiento de la población», «La expansión comercial y de los transportes» [O]; «La expansión agrícola del siglo XVIII» [Vs].

En ocasiones los epígrafes indican la noción de «cambio» recurriendo al adjetivo «nuevo» antepuesto al sustantivo: «Las nuevas fuentes de energía», 
«Las nuevas industrias» [C]; «Nuevas fuentes de energía» [O]; «Nuevas ramas industriales» [O]; «Introducción de nuevos cultivos» [SM]; «Aplicación de nuevas técnicas agrícolas» [SM]; «Una nueva mentalidad» [VS].

\subsection{Verbos de cambio y de proceso}

La principal característica discursiva de los manuales de historia es el uso del pasado, las secuencias narrativas se imponen a las expositivas predominantes en otros tipos de textos académicos. En el caso que nos ocupa, en la descripción de acciones (narración), predominan los verbos que indican cambio y proceso.

\subsubsection{Cambio}

«Descender», «disminuir», «aumentar», «incrementar», «reducir», «mejorar», «cambiar», «convertir», «generalizar», «pasar a», «dejar de»: «La mortalidad descendió»; «disminuyeron las grandes epidemias; «la población aumentó»; "el aumento de la población incrementó la demanda de productos» [A]; «la mortalidad se redujo» [C]; «La ganadería extensiva mejoró» [C]; «Cambiaron las estructuras agrarias» [Vs]; «[esta burguesía] se convirtió en el principal motor de la revolución» [SM]; «El trabajo infantil se generalizó» [SM]; «El sistema de explotación pasó a ser privado» [C]; «La carne dejó de ser un alimento solo al alcance de los más ricos» [Vs].

\subsubsection{Proceso}

- Inicio del proceso o surgimiento

«Iniciar», «introducir», «promover», «aparecer», «desarrollar», «crear», «producir», «nacer», «comenzar»: «Esta transformación se inició», «la población europea inició», «se introdujeron máquinas», «los grandes propietarios ingleses promovieron» [A]; «Hacia 1750 se introdujo el arado de hierro» [Vs]; "A nivel general, entre 1870 y 1914 aparecieron innovaciones tecnológicas y nuevas fuentes de energía, se crearon extraordinarios centros fabriles, se produjo un gran desarrollo del capitalismo y nacieron importantes organizaciones obreras»; «comenzó el uso 
de otras fuentes de energía»; «la industria del automóvil nació...»; «la dinamo apareció en 1866» [o].

- Continuación del proceso

«Mantenerse», «seguir»: «La natalidad se mantuvo alta» [A]: «se siguió utilizando el carbón mineral» [C].

- Fin del proceso o expresión de logro «Alcanzar», «obtener», «acabar con», «desarrollarse»: «El país donde los cambios demográficos alcanzaron mayores proporciones» [A]; «la industria química, que alcanzó gran desarrollo...»[C]; «El comercio exterior británico obtuvo importantes beneficios» [O]; «Las revoluciones liberales acabaron con las propiedades señoriales y comunales [vs]; «se desarrolló la nueva metalurgia» [C].

\subsection{Expresión de causas y efectos}

3.3.1. Verbos y perífrasis verbales que indican relaciones causales

«Ocasionar», «hacer que», «aumentar» (transitivo), «provocar», «dar lugar a», «convertir en», «ser» (la causa de X es Y), «debido a», «proporcionar», «producir», «contribuir a»: «[transformación en la industria] que ocasionó el paso de una economía agraria a otra industrial» [A]; «La elevada natalidad hizo que la población [...] creciera» [C]; «Este crecimiento de la población aumentó la demanda de productos, la creación de un mercado interior, y provocó que la mano de obra sobrante en el campo fuera a trabajar a las fábricas» [C]; «los nuevos inventos que darían lugar a importantes industrias» [C]; «La Revolución Industrial consistió en un conjunto de profundos cambios que convirtieron una economía exclusivamente agrícola y comercial en una economía industrializada» [o]; «La causa de este incremento fue la disminución de la mortalidad, debido a las mejoras en la alimentación y la higiene» [o]; «El aumento demográfico ocasionó una mayor demanda de productos agrícolas e industriales y proporcionó una mano de obra numerosa y barata a la industria» [o]; «El desarrollo del comercio produjo un excedente económico que contribuyó a financiar la expansión industrial» [o]. 


\subsubsection{Consecuencia}

- De esta forma «De esta forma, aumentó el número de cabezas de ganado» [Vs].

- Como resultado de «Como resultado de todo esto, entre 1700 y 1800 la producción del sector primario se incrementó considerablemente» [Vs].

\subsubsection{Conectores causales}

\section{- Gracias a}

«Gracias a» es el conector causal más utilizado en los cinco manuales. Nos limitaremos a reproducir algunos pocos ejemplos de cada libro, aunque su profusión es grandísima: «La natalidad se mantuvo alta, pero la mortalidad descendió gracias a la mejor alimentación, motivada por los procesos agrarios»[A]; "Todo ello se pudo conseguir gracias a la aplicación de los adelantos técnicos de la industria» [C]; «Estos cambios fueron posibles gracias a la aplicación de nuevas máquinas en la industria...» [o]; «Esta burguesía enriquecida gracias a las actividades comerciales y financieras...» [SM]; «La Revolución Industrial comenzó a mediados del siglo XVIII en Europa gracias al uso de nuevas técnicas, fuentes de energía...» [vs].

- Por

«Las transformaciones agrarias [...] se vieron impulsadas por el aumento de la demanda de alimentos» [A]; «Por todo ello, aparecieron las primeras asociaciones en defensa y protección del mundo obrero» [SM].

\subsection{Marcadores de orden}

A veces encontramos marcadores textuales que indican el orden de los cambios en el proceso de transformación que se narra. Así, cuando se habla de las innovaciones en la industria textil, en el libro de Anaya aparecen en negrita los marcadores y los cambios: «Al principio, se adoptaron máquinas hidráulicas», «Finalmente, se emplearon máquinas de vapor». Con ello se facilita de manera visual, a través de la tipografía resaltada, que el alumno pueda seguir 
los hitos que marcan las fases del proceso de transformación y pueda subrayar o esquematizar en su cuaderno estas ideas clave.

\section{Nociones generales y competencia comunicativa: el acceso discursivo a los contenidos históricos}

Una vez inventariadas las formas lingüísticas que textualizan en los manuales estudiados los conceptos temporales asociados al saber histórico a que se refiere Blanco Rebollo en su estudio, nos proponemos vincular estos conceptos con el término noción de la didáctica de las lenguas en relación con el método nocional-funcional. El Plan curricular del Instituto Cervantes, que fija para la lengua española las nociones y las funciones comunicativas, los géneros discursivos y las estructuras gramaticales usados en los diferentes niveles de referencia del Marco europeo de referencia para las lenguas, puede ser un buen punto de partida para delimitar las estructuras gramaticales que aparecen en los libros de texto de Ciencias sociales y para definir las necesidades comunicativas de los estudiantes de Educación Secundaria (en nuestro caso, de cuarto curso) en relación con los textos de historia. El Plan curricular del Instituto Cervantes. Niveles de referencia para el español se inspira en los niveles de descripción para el inglés: Waystage (nivel A2 del Marco europea), Threshold (B1) y Vantage (B2); y para el francés: Niveau Al pour le français (utilisateurapprenant élémentaire): un référentiel y Le niveau B2 pour le français. ${ }^{2}$

Las funciones son las intenciones comunicativas del hablante («identificarse», por ejemplo); las nociones hacen referencia a los «contenidos que tienen que ver con el significado», se trataría de categorías de «carácter semánticogramatical», concebidas como conceptos descriptivos generales. Las nociones serían, pues, «los conceptos a los que el hablante se refiere al realizar un acto de habla». Los descriptores de los niveles de referencia distinguen entre no-

2. La referencias completas son las siguientes: J. A. van Ek / J. L. M., Waystage 1990, Nueva York, Cambridge University Press, 1991; J. A. van Ek / J. L. M., Threshold 1990, Cambridge, Cambridge University Press, 1998; J. A. van Ek / J. L. M., Vantage, Cambridge University Press, Nueva York, 2001; Jean Claude Beacco et alii, Le niveau B2 pour le français (utilisateurapprenant indépendant): un référentiel, París, Didier, 2004; Jean Claude Beacco et alii, Niveau A1 pour le français (utilisateur-apprenant élémentaire): un référentiel, París, Didier, 2007. En catalán existe también la descripción del nivel umbral (B1): Marta Mas / Joan Melcion, Nivell llindar per a la llengua catalana, Barcelona, Departament de Cultura, 1999; a partir de esta descripción del nivel, se hizo una adaptación para estudiantes, muy útil para el tema que nos ocupa: Mercè Belart / Lluïsa Rancé, Nivell llindar per a escolars de 8 a 14 anys, Barcelona, Departament d'Ensenyament-Generalitat de Catalunya, 1995. 
ciones generales, que son las que ahora nos interesan, y las especificas. Las nociones generales son aquellas que un hablante puede necesitar en cualquier contexto comunicativo y se refieren a conceptos abstractos. Las nociones generales se organizan en categorías (nociones espaciales, por ejemplo) y en subcategorías: localización, posición, distancia, movimiento, etc. (Instituto Cervantes, 2006).

Por otra parte el Plan determina que los libros de texto y, en general, los géneros discursivos y productos textuales del ámbito académico serían accesibles a los usuarios a partir de un nivel B2 de competencia y hasta el C2:

B2

- Libros de texto relacionados con la propia especialidad (recepción)

- Trabajos académicos (recepción)

- Trabajos de clase de extensión media (composición escrita) (producción)

C1

- Libros de texto (recepción)

- Trabajos de clase (recepción) (producción) (composición escrita)

$\mathrm{C} 2$

- Trabajos académicos (ensayos, memorias, tesis) (recepción) (producción)

Ahora vamos a clasificar las formas lingüísticas referidas a las nociones de cambio y de causalidad que hemos inventariado en los manuales según las categorías y las subcategorías del Plan curricular y las vamos a poner al lado de las formas recogidas en el nivel de referencia para el inglés Vantage (B2) (Ek/Trim, 2001). 
Plan curricular del Instituto Cervantes

Nociones generales

4.3 Aspectos de desarrollo $(\mathrm{C} 1, \mathrm{C} 2)$

4.3.7 Inicio

iniciar, introducir, promover, aparecer, desa-

rrollar, crear, producir, nacer, comenzar

aparición
Vantage (B2)

General notions

3. Temporal

\subsection{Commencement}

to begin, to start, to go
4.3.8 Finalización

alcanzar, obtener, acabar con, desarrollarse
3.25. Cessation

(to) end, (to) finish, (to) stop

\subsubsection{Continuación}

mantenerse, seguir

\subsection{Continuity}

to continue, to go on

3.26 Stability

to remain, to stay, to keep

3.4. Duration

to continue, to go on, to last

\subsubsection{Cambio}

descender, disminuir, aumentar, incrementar, reducir, mejorar, cambiar, convertir, generalizar, pasar a, dejar de expansión, ampliación, aumento, crecimiento, incremento, avance, mejora, disminución

\subsection{Change, transition}

to become, (to) change, to get, to interrump, to turn, to vary suddenly

7. Relational

ocasionar, hacer que, aumentar (transitivo), provocar, dar lugar a, convertir en, ser (la causa de $\mathrm{X}$ es $\mathrm{Y}$ ), proporcionar, producir, contribuir a debido a, gracias a, por $^{3}$

\subsubsection{Causative}

to have, to make

\subsubsection{Cause}

because, as, due to, because of

3. El Plan curricular no incluye la categoría «relación» en su inventario. «Gracias a» se recoge en el apartado 15.3. «Oraciones subordinadas adverbiales», subapartado «Nexos y conectores de causalidad» del capítulo «Gramática» (nivel C1); «debido a» en el mismo subapartado del nivel B2. Algunos verbos que hemos vinculado a la noción de causa se podrían clasificar también en la noción de «cambio»; en otros casos el Plan los inventaría bajo nociones generales distintas, este es el caso de «ocasionar» $\mathrm{y}$ «provocar» que se adscriben a la noción general 1. «Nociones existenciales» $\rightarrow 1.1$ Existencia, inexistencia» (nivel B2). 


\section{Conclusiones}

Como conclusión podemos afirmar que el conocimiento de las características discursivas que son el resultado de la textualización del saber sabio en la segunda transposición didáctica, resulta de vital importancia para mejorar el acceso a los contenidos curriculares por parte del alumno: la comprensión lectora de textos de tema histórico propios del ámbito académico y la comunicación oral y escrita de discursos expositivos de las ciencias sociales. El análisis discursivo debería facilitar también el diseño de tareas competenciales en las que se trabaje la competencia en comunicación lingüística desde el área de ciencias sociales (y por extensión desde el resto de las áreas).

Por otro lado, el análisis discursivo de los textos escolares vinculado a las nociones y las estructuras gramaticales clasificadas por niveles de dominio de las lenguas con respecto al Marco común europeo para las lenguas nos permite componer textos didácticos, traducirlos y adaptarlos manteniendo un control sobre su nivel de dificultad lingüística y, al mismo tiempo, sobre el proceso de aprendizaje.

En el proceso de desarrollo de la enseñanza plurilingüe en que se encuentra el sistema educativo español, el conocimiento de las estructuras discursivas vinculadas a determinadas nociones y funciones y a los niveles de referencia del Marco común europeo facilitaría el acceso a los contenidos curriculares a través de las diferentes lenguas vehiculares en el método de Tratamiento integrado de lenguas y contenidos (TILC), también conocido como Aprendizaje integrado de contenidos y lenguas extranjeras (AICLE o CLIL). En este caso, en que el acceso al saber histórico se realiza a través de una segunda lengua (L2), un mejor conocimiento de la estructura lingüística de los textos escolares nos ayuda en la creación, adaptación y traducción de materiales de calidad en diferentes lenguas. Así vemos que habría que adaptar los textos didácticos a unos niveles de dificultad lingüística inferiores a un nivel B2 y ello sin desvirtuar el contenido en el proceso de transposición didáctica-textualización.

Por otra parte, el contraste de estructuras lingüísticas de diferentes lenguas vinculadas a nociones comunes facilita el control lingüístico en la modalidad de AICLE conocida como language across the curriculum en que la lengua se aprende al mismo tiempo que los contenidos. También sirve de referencia en aquellas otras modalidades de AICLE en que la asignatura de lengua presta apoyo lingüístico a la asignatura que vehicula los contenidos curriculares en una L2. De esta manera sabemos en cada momento qué estructuras lingüísticas hay que reforzar en la clase de lengua para facilitar el acceso a los contenidos en la clase del área correspondiente. 
Se trata, en suma, de enseñar a dominar el discurso histórico para comprender los textos de historia y poder comunicar contenidos de historia. En esta línea de trabajo nos puede servir el método nocional-funcional de enseñanza de las lenguas: observar cómo se fijan lingüísticamente nociones generales propias del discurso histórico como «causa», «consecuencia», «cambio», puede ser de gran ayuda para los profesores de ciencias sociales. El Plan curricular del Instituto Cervantes, que fija para la lengua española las nociones y las funciones comunicativas usadas en los diferentes niveles del Marco común europeo de referencia para las lenguas podría ser un punto de partida.

\section{Referencias bibliográficas}

Blanco Rebollo, Á. (2008): «La temporalidad histórica en los libros de texto» en Burguera, J.; J. C. Luque; C. Fuentes (2008): 55-61.

Bronckart, J. P.; I. Plazaola (2000): «La transposició didàctica. Història i perspectives d'una problemàtica fundacional» en CAMPS, A.; M. FERRER (coords.): Gramàtica a l'aula, Barcelona, Graó, 39-63.

Burguera, J. (2002): «Los libros de historia del bachillerato en Cataluña: análisis de los contenidos», Íber, núm. 33 (julio-agosto-septiembre): 95-108.

Burguera, J.; J. C. Luque; C. Fuentes (2008): "Anàlisi comparativa del currículum de l'ESO i la seva concreció en els llibres de text» en Els llibres de text $i$ l'ensenyament de la Història. Record d'en Jordi Burguera Gómez, Universitat de Barcelona, Departament de Didàctica de les Ciències Socials, 49-53.

Chevallard, Y. (1991): La transposición didáctica. Del saber sabio al saber enseñado, Buenos Aires, Aique Grupo Editor, 2000.

DEWEY, J. (1925): Experience \& Education, Edición Kindle.

Eк, J. A.; J. L. M. Trim (2001): Vantage, Nueva York, Cambridge University Press.

Gimeno SACRISTÁn, J. (1988): El curriculum: una reflexión sobre la práctica, Madrid, Ediciones Morata.

InSTITUTO CERVANTES (2006): Plan curricular del Instituto Cervantes. Niveles de referencia para el español, Madrid, Instituto Cervantes-Biblioteca Nueva. [Disponible en línea: http:/cvc.cervantes.es/Ensenanza/Biblioteca_Ele/plan_curricular/. Citada 13 marzo 2014].

SAlvador, V. (2005): "Combinatòria lèxica i discurs acadèmic: aplicació a l'anàlisi de manuals d'ensenyament mitjà», Articles de Didàctica de la Llengua i de la Literatura, núm. 36: 75-87. 
Stenhouse, L. (1985): La investigación como base de la enseñanza, Madrid, Ediciones Morata, 1998.

VALLS, R. (2007): «Fuentes y referentes del saber escolar: los actuales manuales escolares (de historia) y criterios para su análisis y valoración» en Ávila RuIz, R. M. et al., (eds.) (2007): Las competencias profesionales para la enseñanza-aprendizaje de las Ciencias Sociales ante el reto europeo y la globalización, Bilbao, Asociación Universitaria de Profesores de Didáctica de las Ciencias Sociales, 499-511.

\section{Decretos curriculares}

LoE, Decreto de enseñanzas mínimas correspondientes a la Educación Secundaria Obligatoria (Real Decreto 1631/2006, de 29 de diciembre), BOE (5-1-2007).

Decret 112/2007, de 20 de juliol, del Consell, pel qual s'establix el currículum de l'Educació Secundària Obligatòria a la Comunitat Valenciana. (DOGV, 24-7-2007).

\section{Libros de texto analizados}

AA. Vv.: Historia $4 .^{\circ}$ ESO. Comunidad Valenciana, Proyecto Los Caminos del Saber, Santillana Educación/Edicions Voramar, Picanya, 2012. IsBN 97884-9807-627-1 [Sv].

Burgos, M.; M. C. Muñoz-Delgado: Historia. Ciencias Sociales 4 Educación secundaria C. Valenciana, Madrid, Grupo Anaya, 2012. IsBN 978-84678-2515-2 [A].

Fernández Bulete, V.: Ciencias sociales. Historia $4 .^{\circ}$ ESO, Proyecto Conecta 2.0, Ediciones SM, 2011. IsBN 978-84-675-4848-8 [SM].

Gorgues, R. et al.: Historia $4 .^{\circ}$ ESO, Valencia, Castellnou Editorial Valenciana, 2008. IsBN 978-84-8345-316-2 [C].

Díaz Rubiano, M. et al.: Historia $4 .^{\circ}$ Secundaria, Edición Comunidad Valenciana, Proyecto Ánfora, Ariz-Basauri, Madrid, Oxford Educación, 2008. ISBN 978-84-673-4287-1 [o]. 